\title{
ON THE AUTOMATICITY OF THE HANKEL DETERMINANTS OF A FAMILY OF AUTOMATIC SEQUENCES
}

\author{
YINING HU AND GUONIU WEI-HAN
}

\begin{abstract}
Hankel determinants and automatic sequences are two classical subjects widely studied in Mathematics and Theoretical Computer Science. However, these two topics were considered totally independently, until in 1998, when Allouche, Peyrière, Wen and Wen proved that all the Hankel determinants of the Thue-Morse sequence are nonzero. This property allowed Bugeaud to prove that the irrationality exponents of the Thue-Morse-Mahler numbers are exactly 2. Since then, the Hankel determinants of several other automatic sequences, in particular, the paperfolding sequence, the Stern sequence, the period-doubling sequence, are studied by Coons, Vrbik, Guo, Wu, Wen, Bugeaud, Fu, Han, Fokkink, Kraaikamp, and Shallit. On the other hand, it is known that the Hankel determinants of a rational power series are ultimately zero, and the Hankel determinants of a quadratic power series over finite fields are ultimately periodic. It is therefore natural to ask if we can obtain similar results about the Hankel determinants of algebraic series. In the present paper, we provide a partial answer to this question by establishing the automaticity of the reduced Hankel determinants modulo 2 of a family of automatic sequences. As an application of our result, we give upper bounds for the irratoinality exponent of a family of automatic numbers.
\end{abstract}

\section{INTRODUCTION}

The present paper deals with two classical objects in Mathematics and Theoretical Computer Science, namely, Hankel determinants and automatic sequences. For each infinite sequence $\mathbf{c}=\left(c_{j}\right)_{j \geq 0}$ and each nonnegative integer $n$ the Hankel determinant of order $n$ of the sequence $\mathbf{c}$ is defined by

$$
H_{n}(\mathbf{c}):=\left|\begin{array}{cccc}
c_{0} & c_{1} & \cdots & c_{n-1} \\
c_{1} & c_{2} & \cdots & c_{n} \\
\vdots & \vdots & \ddots & \vdots \\
c_{n-1} & c_{n} & \cdots & c_{2 n-2}
\end{array}\right| .
$$

We also speak of the Hankel determinants of the power series $\tilde{\mathbf{c}}(x)=\sum_{k \geq 0} c_{j} x^{j}$ and write $H_{n}(\tilde{\mathbf{c}}(x))=H_{n}(\mathbf{c})$. The Hankel determinants are widely studied in Mathematics and, in several cases, can be evaluated by basic determinant manipulation, $L U$-decomposition, or Jacobi continued fraction (see, e.g., [20, 21, 12]). In this article, we consider the reduced Hankel determinants of \pm 1 -sequences, defined as $H_{n}(\mathbf{c}) / 2^{n-1}$, since the Hankel determinant of order $n$ of a \pm 1 -sequence is always divisible by $2^{n-1}$.

Date: August 12, 2018.

2010 Mathematics Subject Classification. 05A05, 11B50, 11B85, 11C20 11J72, 11J82.

Key words and phrases. automatic sequence, Hankel determinant, irrationality exponent. 
On the other hand, a sequence is said to be d-automatic if it can be generated by a $d$-DFAO (deterministic finite automaton with output) [3]. For an integer $d \geq 2$, a $d$-DFAO is defined to be a 6 -tuple

$$
M=\left(Q, \Sigma, \delta, q_{0}, \Delta, \tau\right)
$$

where $Q$ is the set of states with $q_{0} \in Q$ being the initial state, $\Sigma=\{0,1, \ldots, d-1\}$ the input alphabet, $\delta: Q \times \Sigma \rightarrow Q$ the transition function, $\Delta$ the output alphabet, and $\tau: Q \rightarrow \Delta$ the output function. The $d$-DFAO $M$ generates a sequence $\left(c_{n}\right)_{n \geq 0}$ in the following way: for each non-negative integer $n$, the base- $d$ expansion of $n$ is read by $M$ from right to left starting from the initial state $q_{0}$, and the automaton moves from state to state according to its transition funciton $\delta$. When the end of the string is reached, the automaton halts in a state $q$, and the automaton outputs the symbol $c_{n}=\tau(q)$. In practice, an automaton is often presented as a multigraph with states as vertices and the transition function as directed edges labelled by the input alphabet.

Here is an example of a 2-automaton:

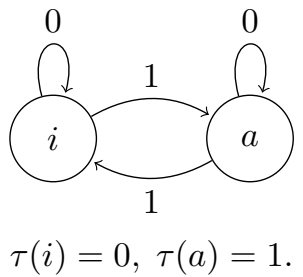

The sequence $(t(n))_{n}$ generated by this automaton is the Thue-Morse sequence whose first terms are $0110100110010110 \cdots$.

An equivalent definition for a sequence $\mathbf{u}$ to be $d$-automatic is that the $d$-kernel of $\mathbf{u}$, defined as

$$
\left\{\left(u\left(d^{k} n+j\right)\right)_{n \geq 0} \mid k \in \mathbb{N}, 0 \leq j \leq d^{k}-1\right\},
$$

is finite [11, Prop. V.3.3], 8. If we let $\Lambda_{i}^{(d)}$ denote the operator that sends a sequence $(u(n))_{n \geq 0}$ to its subsequence $(u(d n+i))_{n \geq 0}$, then the $d$-kernel can be defined alternatively as the smallest set containing $\mathbf{u}$ that is stable under $\Lambda_{i}^{(d)}$ for $0 \leq i<d-1$. We write $\Lambda_{i}$ instead of $\Lambda_{i}^{(d)}$ when the value of $d$ is clear from the context.

Automatic sequences arise naturally in various contexts. The study of automatic sequences lies at the interface of number theory, combinatorics on words, dynamic system, logic and theoretical computer science. We refer the readers to 3 for a comprehensive exposition of the subejct. One fundamental result is the link between automatic sequences and algebraicity in positive characteristic, discovered by Christol, Kamae, Mendès France and Rauzy 1980 [7. They proved that a series in $\mathbb{F}_{q}[[x]]$ is algebraic over $\mathbb{F}_{q}(x)$ if and only if the sequence of its coefficients is $q$-automatic.

Hankel determinants and automatic sequences were considered totally independently, until in 1998, when Allouche, Peyrière, Wen and Wen proved that all the Hankel determinants of the Thue-Morse sequence are nonzero [2]. This property allowed Bugeaud to prove that the irrationality exponents of the Thue-Morse-Mahler numbers are exactly $2[5]$. Since then, the Hankel determinants for several other automatic sequences, in particular, the paperfolding sequence, the Stern sequence, the 
period-doubling sequence, are studied by Coons, Vrbik, Guo, Wu, Wen, Bugeaud, Fu, Han, Fokkink, Kraaikamp, and Shallit [10, 16, 19, 13, 17, 18, 14, 6].

Apart from their link with irrationality exponent, one reason for considering the Hankel determinants of automatic sequences is the following: It is known that a formal power series is rational if and only if its Hankel determinants are ultimately zero [22, p. 5, Kronecker Lemma], and the Hankel determinants of a quadratic power series over finite fields are ultimately periodic 18 . It is therefore reasonable to expect analogous properties from the Hankel determinants of algebraic series.

We now introduce the class of sequences that we will study in this article. Let $d \geq 2$ be a positive integer and $\mathbf{v}=\left(v_{0}, v_{1}, v_{2}, \ldots, v_{d-1}\right)$ a finite \pm 1 -sequence of length $d$ such that $v_{0}=1$. The generating polynomial of $\mathbf{v}$ is denoted by $\tilde{\mathbf{v}}(x)=\sum_{i=0}^{d-1} v_{i} x^{i}$. We consider the \pm 1 -sequence $\mathbf{f}$ defined by the following power series

$$
\tilde{\mathbf{f}}(x):=\Phi(\tilde{\mathbf{v}}(x)):=\prod_{k=0}^{\infty} \tilde{\mathbf{v}}\left(x^{d^{k}}\right)
$$

We see that the $n$-th term of $\mathbf{f}$ is equal to

$$
f_{n}=\prod_{i=1}^{d-1} v_{i}^{s_{d, i}(n)},
$$

where $s_{d, i}(n)$ denotes the number of occurrences of the digit $i$ in the base- $d$ representation of $n$. Thus, the Thue-Morse power series is equal to $\Phi(1-x)=\prod_{k=0}^{\infty}\left(1-x^{2^{k}}\right)$. Since the power series $\tilde{\mathbf{f}}(x)$ satisfies the following functional equation

$$
\tilde{\mathbf{f}}(x)=\tilde{\mathbf{v}}(x) \prod_{k=1}^{\infty} \tilde{\mathbf{v}}\left(x^{d^{k}}\right)=\tilde{\mathbf{v}}(x) \tilde{\mathbf{f}}\left(x^{d}\right),
$$

the sequence $\mathbf{f}$ can also be defined by the recurrence relations

$$
f_{0}=1, \quad f_{d n+i}=v_{i} f_{n} \quad \text { for } n \geq 0 \text { and } 0 \leq i \leq d-1 .
$$

We prove the following Theorem, which we will use in Section 4 to obtain upper bounds for the irrationality exponent of a family of automatic numbers.

Theorem 1.1. For each positive integer $d \geq 2$ and each \pm 1 -vector $\mathbf{v}$ of length $d$, the sequence of the reduced Hankel determinants modulo 2 of the sequence $\mathbf{f}$ defined by $\tilde{\mathbf{f}}=\Phi(\tilde{\mathbf{v}}(x))$ is d-automatic.

The proof of Theorem 1.1 is based on some previous works about the Hankel determinants developed by Fu-Han [14, see Section 2, We give two examples of Theorem 1.1 in Section 3 . The generating series of the two sequences are respectively

$$
\prod_{k \geq 0}\left(1+x^{5^{k}}-x^{2 \cdot 5^{k}}-x^{3 \cdot 5^{k}}+x^{4 \cdot 5^{k}}\right)
$$

and

$$
\prod_{k \geq 0}\left(1+x^{4^{k}}-x^{2 \cdot 4^{k}}-x^{3 \cdot 4^{k}}\right) .
$$

By Theorem 1.1 the sequence of the reduced Hankel determinants modulo 2 is 5automatic (resp. 4-automatic). The minimal automata are illustrated in Section 3 , 


\section{Proof of the Main Theorem}

In this section we first recall some previous works developed in [14] in order to explain the meaning of the notions used in the proof of the Main Theorem. Then we prove two general Lemmas. Finally we apply these two Lemmas to the results from [14] to prove the Main Theorem.

2.1. Key notions from previous works. In the proof of the Main Theorem, we will make use of recurrence relations between six sequences $\mathbf{X}, \mathbf{Y}, \mathbf{Z}, \mathbf{U}, \mathbf{V}, \mathbf{W}$. These are notions from the previous works of $\mathrm{Fu}$ and Han 14. In this subsection we recall definitions and results about these sequences. The most important thing to keep in mind for the sake of the proof of the Main Theorem is that $\mathbf{Z}$ is the sequence of reduced Hankel determinants modulo 2.

The following two disjoint infinite sets of integers $J$ and $K$ associated with $\mathbf{v}$ play an important role in the proof of Theorem 1.1

Definition 2.1. Let $P=\left\{1 \leq i \leq d-1 \mid v_{i-1} \neq v_{i}\right\}$ and $Q=\{1 \leq i \leq d-1 \mid$ $\left.v_{i-1}=v_{i}\right\}$. If $v_{d-1}=-1$, define

$$
\begin{aligned}
J= & \left\{(d n+p) d^{2 k}-1 \mid n, k \in \mathbb{N}, p \in P\right\} \\
& \bigcup\left\{(d n+q) d^{2 k+1}-1 \mid n, k \in \mathbb{N}, q \in Q\right\}, \\
K= & \left\{(d n+q) d^{2 k}-1 \mid n, k \in \mathbb{N}, q \in Q\right\} \\
& \bigcup\left\{(d n+p) d^{2 k+1}-1 \mid n, k \in \mathbb{N}, p \in P\right\} .
\end{aligned}
$$

If $v_{d-1}=1$, define

$$
\begin{aligned}
J & =\left\{(d n+p) d^{k}-1 \mid n, k \in \mathbb{N}, p \in P\right\}, \\
K & =\left\{(d n+q) d^{k}-1 \mid n, k \in \mathbb{N}, q \in Q\right\} .
\end{aligned}
$$

Let $\mathfrak{S}_{m}=\mathfrak{S}_{\{0,1, \ldots, m-1\}}$ be the set of all permutations on $\{0,1, \ldots, m-1\}$. For $m \geq \ell \geq 0$ let $\mathfrak{J}_{m, \ell}\left(\operatorname{resp} . \mathfrak{K}_{m, \ell}\right)$ be the set of all permutations $\sigma=\sigma_{0} \sigma_{1} \cdots \sigma_{m-1} \in$ $\mathfrak{S}_{m}$ such that $i+\sigma_{i} \in J$ (resp. $i+\sigma_{i} \in K$ ) for $i \in\{0,1, \ldots, m-1\} \backslash\{\ell\}$. Write:

$$
j_{m, \ell}:=\# \mathfrak{J}_{m, \ell}, \quad k_{m, \ell}:=\# \mathfrak{K}_{m, \ell}
$$

Definition 2.2. We define the following sequences taking values in $\mathbb{Z} / 2 \mathbb{Z}$ :

$$
\begin{aligned}
X_{n} & :=\sum_{i=0}^{n-1} j_{n, i} \bmod 2, \quad Y_{n}:=j_{n, n} \bmod 2, \quad Z_{n}:=j_{n, n-1} \bmod 2, \\
U_{n} & :=\sum_{i=0}^{n-1} k_{n, i} \bmod 2, \quad V_{n}:=k_{n, n} \bmod 2, \quad W_{n}:=k_{n, n-1} \quad \bmod 2,
\end{aligned}
$$

for $n \geq 1$, and $X_{0}:=0, Y_{0}:=1, Z_{0}:=0, U_{0}:=0, V_{0}:=1, W_{0}:=0$.

Notice that if $\ell=m$, then $\{0,1, \ldots, m-1\} \backslash\{\ell\}=\{0,1, \ldots, m-1\}$, so that $j_{m, m}$ (resp. $\left.k_{m, m}\right)$ is the number of permutations $\sigma \in \mathfrak{S}_{m}$ such that $i+\sigma(i) \in J$ (resp. $\in K$ ) for all $i$. From the definitions above it is easy to see that $\mathbb{N}=J \cup K$ and we have the following lemma.

Lemma 2.1. For each $t \geq 0$ the integer $\delta_{t}:=\left|\left(f_{t}-f_{t+1}\right) / 2\right|$ is equal to 1 if and only if $t$ is in $J$. 
For the proof of the the following theorem, we use the same method as in the proof of Theorem 2.2 in 15 .

Theorem 2.2. Let $\mathbf{v}$ be $a \pm 1$-sequence of length $d$ with $v_{0}=1$. The sequence $\mathbf{f}$ and the set $J$ associated with $\mathbf{v}$ are defined by (1.2) and Definition 2.1 respectively. Then, the reduced Hankel determinant

$$
H_{m}(\mathbf{f}) / 2^{m-1} \equiv Z_{m} \quad(\bmod 2) .
$$

Proof. Let $m$ be a positive integer. By means of elementary transformations the Hankel determinant $H_{m}(\mathbf{f})$ is equal to

$$
\begin{aligned}
H_{m}(\mathbf{f}) & =\left|\begin{array}{cccc}
f_{0} & f_{1} & \cdots & f_{m-1} \\
f_{1} & f_{2} & \cdots & f_{m} \\
\vdots & \vdots & \ddots & \vdots \\
f_{m-1} & f_{m} & \cdots & f_{2 m-2}
\end{array}\right| \\
& =2^{m-1} \times\left|\begin{array}{ccccc}
\frac{f_{0}-f_{1}}{2} & \frac{f_{1}-f_{2}}{2} & \cdots & \frac{f_{m-2}-f_{m-1}}{2} & f_{m-1} \\
\frac{f_{1}-f_{2}}{2} & \frac{f_{2}-f_{3}}{2} & \cdots & \frac{f_{m-1}-f_{m}}{2} & f_{m} \\
\vdots & \vdots & \ddots & \vdots & \vdots \\
\frac{f_{m-1}-f_{m}}{2} & \frac{f_{m}-f_{m+1}}{2} & \cdots & \frac{f_{2 m-3}-f_{2 m-2}}{2} & f_{2 m-2}
\end{array}\right| .
\end{aligned}
$$

By Lemma 2.1, the reduced Hankel determinant is congruent modulo 2 to

$$
\frac{H_{m}(\mathbf{f})}{2^{m-1}} \equiv\left|\begin{array}{ccccc}
\delta_{0} & \delta_{1} & \cdots & \delta_{m-2} & 1 \\
\delta_{1} & \delta_{2} & \cdots & \delta_{m-1} & 1 \\
\vdots & \vdots & \ddots & \vdots & \vdots \\
\delta_{m-1} & \delta_{m} & \cdots & \delta_{2 m-3} & 1
\end{array}\right| \quad(\bmod 2)
$$

By the very definition of a determinant or the Leibniz formula, the determinant occurring on the right-hand side of the congruence (2.1) is equal to

$$
S_{m}:=\sum_{\sigma \in \mathfrak{S}_{m}}(-1)^{\operatorname{inv}(\sigma)} \delta_{0+\sigma_{0}} \delta_{1+\sigma_{1}} \cdots \delta_{m-2+\sigma_{m-2}},
$$

where $\operatorname{inv}(\sigma)$ is the number of inversions of the permutation $\sigma$. By Lemma 2.1 the product $\delta_{0+\sigma_{0}} \delta_{1+\sigma_{1}} \cdots \delta_{m-2+\sigma_{m-2}}$ is equal to 1 if $i+\sigma_{i} \in J$ for $i=0,1, \ldots, m-2$, and to 0 otherwise. Hence, the summation $S_{m}$ is congruent modulo 2 to the number of permutations $\sigma \in \mathfrak{S}_{m}$ such that $i+\sigma_{i} \in J$ for all $i=0,1, \ldots, m-2$, which is exactly $Z_{m}$ by Definition 2.2 .

In 14] an algorithm was described for finding and also proving a list of recurrence relations between $X_{n}, Y_{n}, Z_{n}, U_{n}, V_{n}, W_{n}$. The program Apwen. py is an implementation of this algorithm in Python. Using these relations, Fu-Han successfully proved that several sequences are Apwenian (i.e., the reduced Hankel determinants $Z_{m}$ are odd for all $m \in \mathbb{N}$ ).

2.2. Two general Lemmas. In this subsection we establish two general Lemmas that will be used in the proof.

For a sequence $\mathbf{u}$, we let $\sigma(\mathbf{u})$ denote the shifted sequence $\sigma(\mathbf{u}): n \mapsto u(n+1)$. Let $S$ be a set of sequences, we let $\sigma(S)$ denote the set $\{\sigma(\mathbf{u}) \mid \mathbf{u} \in S\}$ and $\Lambda(S)$ the set $\left\{\Lambda_{j}(\mathbf{u}) \mid j=0, \ldots, d-1, \mathbf{u} \in S\right\}$. If $S$ is a set of sequences over $\mathbb{Z} / 2 \mathbb{Z}$, we let $\mathfrak{A}(S)$ denote the $\mathbb{Z} / 2 \mathbb{Z}$-algebra generated by elements of $S$. 
Lemma 2.3. Let $S$ be a set of sequences over $\mathbb{Z} / 2 \mathbb{Z}$, if $\Lambda(S) \subseteq \mathfrak{A}(S \cup \sigma(S))$, then $\Lambda\left(\mathfrak{A}\left(S \cup \sigma(S) \cup \sigma^{2}(S)\right)\right) \subseteq \mathfrak{A}\left(S \cup \sigma(S) \cup \sigma^{2}(S)\right)$.

Proof. Since $\Lambda\left(\mathfrak{A}\left(S \cup \sigma(S) \cup \sigma^{2}(S)\right)\right) \subseteq \mathfrak{A}\left(\Lambda\left(S \cup \sigma(S) \cup \sigma^{2}(S)\right)\right)$, we only need to show that $\Lambda\left(S \cup \sigma(S) \cup \sigma^{2}(S)\right) \subseteq \mathfrak{A}\left(S \cup \sigma(S) \cup \sigma^{2}(S)\right)$.

By assumption, $\Lambda(S) \subseteq \mathfrak{A}(S \cup \sigma S) \subseteq \mathfrak{A}\left(S \cup \sigma(S) \cup \sigma^{2}(S)\right)$.

To show that $\Lambda(\sigma(S)) \subseteq \mathfrak{A}\left(S \cup \sigma(S) \cup \sigma^{2}(S)\right)$, let $\mathbf{u}$ be an element of $S$. For $j=0, \ldots, d-2$, we have $\Lambda_{j}(\sigma(\mathbf{u}))=\Lambda_{j+1}(\mathbf{u})$. For $j=d-1$, we have $\Lambda_{j}(\sigma(\mathbf{u}))=$ $\sigma\left(\Lambda_{0}(\mathbf{u})\right)$. Since $\Lambda_{0}(\mathbf{u}) \in \mathfrak{A}(S \cup \sigma(S))$ by assumption, $\sigma\left(\Lambda_{0}(\mathbf{u})\right) \in \sigma(\mathfrak{A}(S \cup \sigma(S)))=$ $\mathfrak{A}\left(\sigma(S) \cup \sigma^{2}(S)\right) \subseteq \mathfrak{A}\left(S \cup \sigma(S) \cup \sigma^{2}(S)\right)$.

To show that $\Lambda\left(\sigma^{2}(S)\right) \subseteq \mathfrak{A}\left(S \cup \sigma(S) \cup \sigma^{2}(S)\right)$, let $\mathbf{u}$ be an element of $S$. For $j=0, \ldots, d-3$, we have $\Lambda_{j}\left(\sigma^{2}(\mathbf{u})\right)=\Lambda_{j+2}(\mathbf{u})$. Besides, $\Lambda_{d-2}\left(\sigma^{2}(\mathbf{u})\right)=\sigma\left(\Lambda_{0}(\mathbf{u})\right)$ and $\Lambda_{d-1}\left(\sigma^{2}(\mathbf{u})\right)=\sigma\left(\Lambda_{1}(\mathbf{u})\right)$. These are elements of $\mathfrak{A}\left(S \cup \sigma(S) \cup \sigma^{2}(S)\right.$ for the same reason as before.

Lemma 2.4. Let $\mathbf{X}^{i}, i=1, \ldots, \ell$ be sequences taking values in a finite ring $R$, and $M_{j}$ be $\ell \times \ell$ matrices with entries in $R$ for $j=0, \ldots, d-1$. We denote by $\mathbf{A}$ the sequence of column vectors $A(n)=\left(X^{1}(n), \ldots, X^{\ell}(n)\right)^{T}$. If

$$
A(d n+j)=M_{j} A(n) \text { for all } j=0, \ldots, d-1 \text {, and all } n \in \mathbb{N},
$$

then $\mathbf{X}^{i}$ is d-automatic.

Proof. As $d$-automaticity is preserved by codings of the output alphabet, we only need to show that the sequence $\mathbf{A}$ over the finite alphabet $R^{\ell}$ is $d$-automatic.

By assumption, for all $j \in\{0, \ldots, d-1\}$, we have

$$
M_{j} A=\Lambda_{j} A \text {. }
$$

Therefore for all $i, j \in\{0, \ldots, d-1\}$,

$$
M_{i} M_{j} A=M_{i} \Lambda_{j} A=\Lambda_{j} M_{i} A=\Lambda_{j} \Lambda_{i} A .
$$

By induction, for any finite word $w=w_{1} \cdots w_{m}$ over the alphabet $\{0, \ldots, d-1\}$,

$$
M_{w_{1}} \cdots M_{w_{m}} A=\Lambda_{w_{m}} \cdots \Lambda_{w_{1}} A .
$$

Therefore the $d$-kernel of $\mathbf{A}$ is the set

$$
\left\{M \mathbf{A} \mid M \in\left\{M_{0}, \ldots, M_{d-1}\right\}^{*}\right\},
$$

which is a subset of the finite set $\left\{M \mathbf{A} \mid M \in M_{\ell \times \ell}(R)\right\}$.

\subsection{The proof.}

Proof of Theorem 1.1. We notice that the algorithm described in [14 is also valid for other \pm 1 -sequences of form (1.2) than Apwenian sequences, and it will produce a list of recurrence relations similar to those found in the two examples in Section 3 . More precisely, if we denote by $S$ the set $\{\mathbf{X}, \mathbf{Y}, \mathbf{Z}, \mathbf{U}, \mathbf{V}, \mathbf{W}\}$ of six sequences introduced in Definition 2.2, then according to Theorem 4.1 of [14], we have recurrence relations that express $\Lambda_{i} \mathbf{S}$ in terms of the $\mathbb{Z} / 2 \mathbb{Z}$-linear combinations of products of elements in $S \cup \sigma(S)$ for all $i=0,1, \ldots, d-1$ and all $\mathbf{S} \in S$. In other words, we have

$$
\Lambda(S) \subseteq \mathfrak{A}(S \cup \sigma(S)) .
$$

According to Lemma 2.3. this implies that $\Lambda\left(\mathfrak{A}\left(S \cup \sigma(S) \cup \sigma^{2}(S)\right)\right) \subseteq \mathfrak{A}(S \cup \sigma(S) \cup$ $\left.\sigma^{2}(S)\right)$ ). Thus if we define $\mathbf{A}$ to be the vector whose components are all the elements in $S \cup \sigma(S) \cup \sigma^{2}(S)$ and all their products, then there exists matrices 
$M_{0}, M_{1}, \ldots, M_{d-1}$ that satisfy the hypothesis of Lemma 2.4. Therefore all the components of $\mathbf{A}$, and in particular $\mathbf{Z}$, are $d$-automatic.

The proofs of Lemma 2.3 and Lemma 2.4 being constructive, we may implement them to find a $d$-DFAO that generates the sequence $\mathbf{Z}$. This is done by the Python program ASHankel.py which takes as input the relations and initial values generated by Apwen.py and computes the minimal $d$-DFAO of $\mathbf{Z}$.

\section{Two EXAmples}

3.1. Example 1. Take $\mathbf{v}=(1,1,-1,-1,1)$ with $d=5$ and $v_{d-1}=1$. Then, the corresponding infinite \pm 1 -sequence $\mathbf{f}$ is equal to

$$
\Phi\left(1+z-z^{2}-z^{3}+z^{4}\right)=\prod_{k \geq 0}\left(1+x^{5^{k}}-x^{2 \cdot 5^{k}}-x^{3 \cdot 5^{k}}+x^{4 \cdot 5^{k}}\right) .
$$

The Python program Apwen.py finds and proves the following recurrences:

$$
\begin{array}{rlrl}
X_{5 n+0} & =X_{n}, & Y_{5 n+0} & =Y_{n}, \\
X_{5 n+1} & =Z_{n+1} Y_{n}, & Y_{5 n+1} & =Z_{n+1} X_{n}\left(Y_{n}+1\right), \\
X_{5 n+2} & =0, & Y_{5 n+2} & =Z_{n+1} Y_{n}, \\
X_{5 n+3} & =0, & Y_{5 n+3} & =Z_{n+1} Y_{n+1}, \\
X_{5 n+4} & =Z_{n+1} Y_{n+1}, & Y_{5 n+4}=Z_{n+1} X_{n+1}\left(Y_{n+1}+1\right), \\
Z_{5 n+0} & =Z_{n}\left(X_{n}+X_{n} Y_{n}+Y_{n}\right), \\
Z_{5 n+1} & =Z_{n+1}\left(X_{n}+X_{n} Y_{n}+Y_{n}\right), \\
Z_{5 n+2} & =Z_{n+1} Y_{n}, \\
Z_{5 n+3} & =0, \\
Z_{5 n+4} & =Z_{n+1} Y_{n+1},
\end{array}
$$

with the initial values:

$$
X_{0}=0, X_{1}=1, Y_{0}=1, Y_{1}=0, Z_{0}=0, Z_{1}=1 .
$$

Recall that $\mathbf{Z}$ is the sequence of the reduced Hankel determinants modulo 2 . By Theorem 1.1 the sequence $\mathbf{Z}$ is 5 -automatic, and its minimal automaton is produced by the program ASHankel . py (See Figure 1).

What the program ASHankel.py does can be decomposed into the following steps: First it expands the given recurrence relations to the relations $\Lambda_{i} \mathbf{A}=M_{i} \mathbf{A}$ for $i=0,1,2,3,4$, where $M_{i}$ are matrices and $\mathbf{A}$ is a vector of dimension 21 whose components are $\mathbf{X}, \mathbf{Y}, \mathbf{Z}, \sigma(\mathbf{X}), \sigma(\mathbf{Y}), \sigma(\mathbf{Z}), \sigma^{2}(\mathbf{X}), \sigma^{2}(\mathbf{Y}), \sigma^{2}(\mathbf{Z})$ and some of their products. Then, by calculating all possible products of the matrices $M_{i}$ for $i=0,1,2,3,4$, it finds a set $K$ of size 43 that maps onto the 5 -kernel of the sequence $\mathbf{A}$, as well as the transition function. After that it reduces the set $K$ and the transition function to a smaller set $S K$ of size 9 and the transition function $\phi: S K \times\{0,1,2,3,4\} \rightarrow S K$ by projecting each product of matrix to the row vector that corresponds to $\mathbf{Z}$. The set $S K$ maps onto the 5 -kernel of $\mathbf{Z}$ naturally. In this way we obtain a 5 -DFAO with $S K$ as the set of states, $\phi$ as the transition function, and as output function the map from $S K$ to the first term of the corresponding sequence in the 5 -kernel of $\mathbf{Z}$. This 5 -DFAO generates the sequence $\mathbf{Z}$. Finally this automaton is converted to the minimal automaton of $\mathbf{Z}$. 


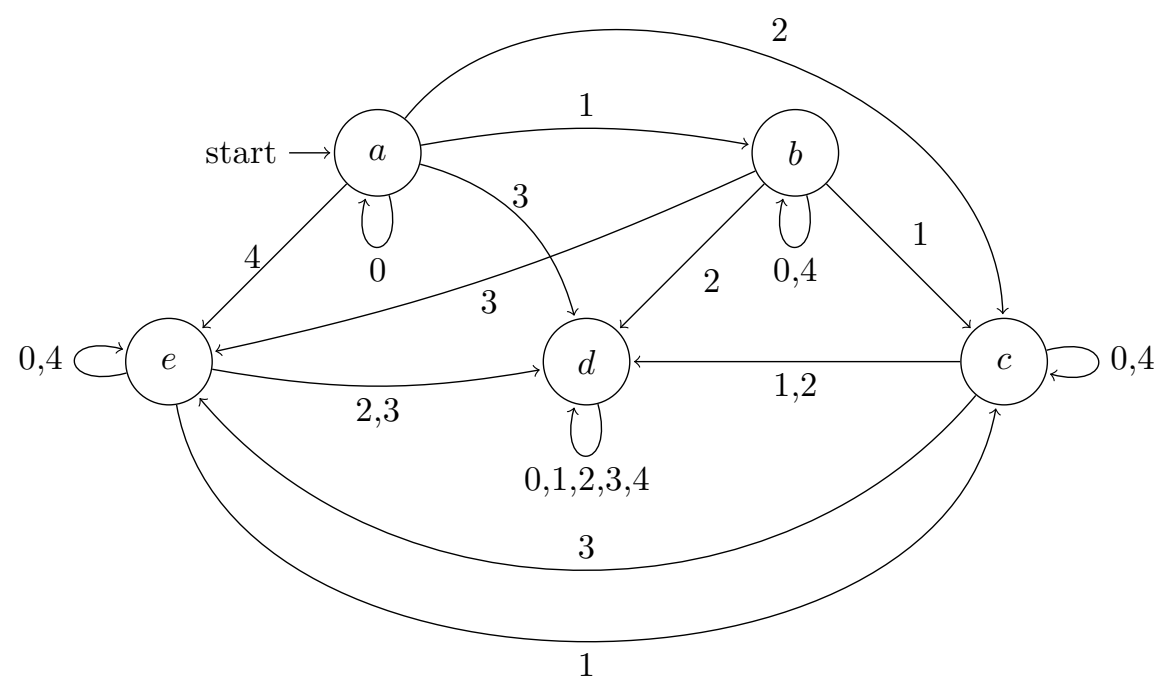

Figure 1. Minimal automaton for Example 1 where the output function is $(a, b, c, d, e) \mapsto(0,1,1,0,0)$.

In the following table, we list the first values of $H_{n}$ and the sequence $\mathbf{u}$ defined by the 5 -DFAO in Figure 1 . We verify that $u_{n}=H_{n} / 2^{n-1} \bmod 2=Z_{n}$.

\begin{tabular}{cccccccccccccccc}
\hline$n$ & 0 & 1 & 2 & 3 & 4 & 5 & 6 & 7 & 8 & 9 & 10 & 11 & 12 & 13 & 14 \\
\hline$H_{n}$ & 1 & 1 & -2 & 0 & 0 & 16 & -32 & -128 & 256 & -1280 & -6656 & 0 & 0 & 0 & 0 \\
$u_{n}$ & 0 & 1 & 1 & 0 & 0 & 1 & 1 & 0 & 0 & 1 & 1 & 0 & 0 & 0 & 0 \\
\hline
\end{tabular}

3.2. Example 2. Take $\mathbf{v}=(1,1,-1,-1)$ with $d=4$ and $v_{d-1}=-1$. Then, the corresponding infinite \pm 1 -sequence $\mathbf{f}$ is equal to

$$
\Phi\left(1+z-z^{2}-z^{3}\right)=\prod_{k \geq 0}\left(1+x^{4^{k}}-x^{2 \cdot 4^{k}}-x^{3 \cdot 4^{k}}\right) .
$$

As explained in [15], we have six sequences $\mathbf{X}, \mathbf{Y}, \mathbf{Z}, \mathbf{U}, \mathbf{V}, \mathbf{W}$, since $v_{d-1}=-1$. Recall that $\mathbf{Z}$ is the sequence of the reduced Hankel determinants modulo 2. The program Apwen. py finds and proves the following recurrences:

$$
\begin{array}{ll}
X_{4 n+0}=0, & U_{4 n+0}=0, \\
X_{4 n+1}=W_{n+1}\left(U_{n}+V_{n}\right), & U_{4 n+1}=Z_{n+1} Y_{n}, \\
X_{4 n+2}=0, & U_{4 n+2}=0, \\
X_{4 n+3}=W_{n+1}\left(U_{n+1}+V_{n+1}\right), & U_{4 n+3}=Z_{n+1} Y_{n+1}, \\
Y_{4 n+0}=U_{n}+V_{n}, & V_{4 n+0}=Y_{n}, \\
Y_{4 n+1}=0, & V_{4 n+1}=Z_{n+1} Y_{n}, \\
Y_{4 n+2}=W_{n+1}, & V_{4 n+2}=Z_{n+1},
\end{array}
$$




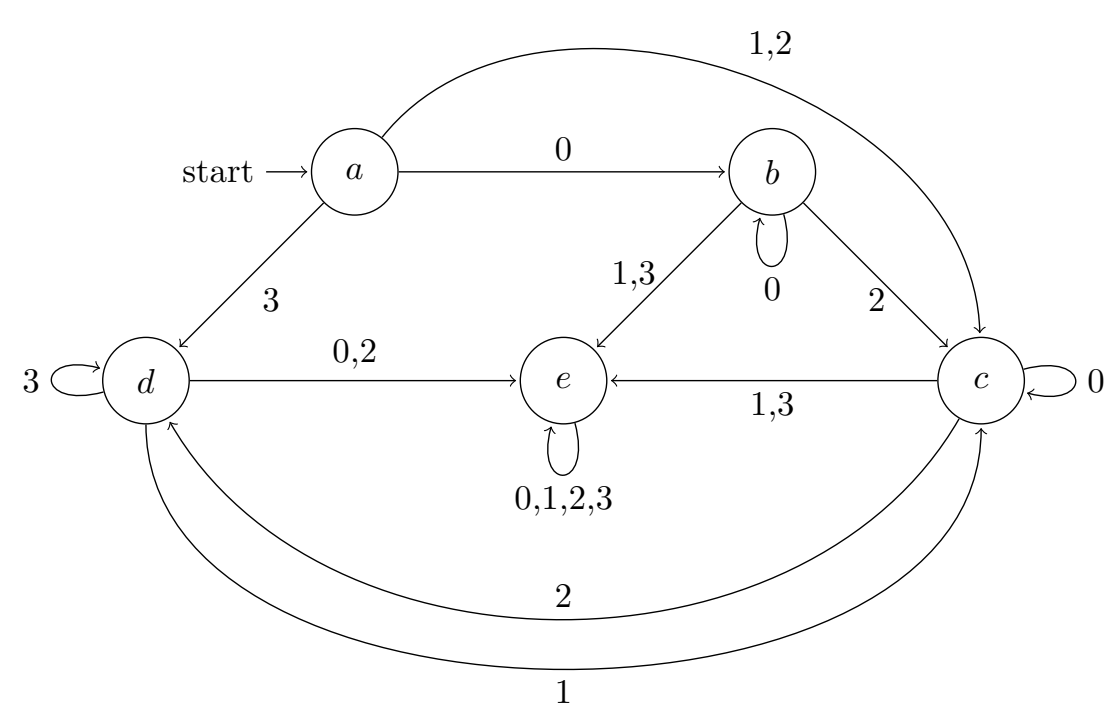

Figure 2. Minimal automaton for Example 2 where the output function: $(a, b, c, d, e) \mapsto(0,0,1,0,0)$.

$$
\begin{aligned}
Y_{4 n+3} & =0, & V_{4 n+3} & =Z_{n+1} Y_{n+1}, \\
Z_{4 n+0} & =W_{n}\left(U_{n}+V_{n}\right), & & W_{4 n+0}=Z_{n} Y_{n}, \\
Z_{4 n+1} & =W_{n+1}\left(U_{n}+V_{n}\right), & W_{4 n+1} & =Z_{n+1} Y_{n}, \\
Z_{4 n+1} & =W_{n+1}\left(U_{n}+V_{n}\right), & W_{4 n+2} & =Z_{n+1} Y_{n}, \\
Z_{4 n+1} & =W_{n+1}\left(U_{n+1}+V_{n+1}\right), & W_{4 n+3} & =Z_{n+1} Y_{n+1},
\end{aligned}
$$

with the initial values:

$$
\begin{aligned}
& X_{0}=0, \quad Y_{0}=1, \quad Z_{0}=0, \quad U_{0}=0, \quad V_{0}=1, \quad W_{0}=0, \\
& X_{1}=1, \quad Y_{1}=0, \quad Z_{1}=1, \quad U_{1}=1, \quad V_{1}=1, \quad W_{1}=1 .
\end{aligned}
$$

By Theorem 1.1, the sequence $\mathbf{Z}$ is 4-automatic. As in the previous example, using the recurrence relations and initial values above, the Python program ASHankel.py computes the minimal automaton of $\mathbf{Z}$ (See Figure 2). In the following table, we list the first values of $H_{n}$ and the sequence $\mathbf{u}$ defined by the 4-DFAO in Figure 2. We verify that $u_{n}=H_{n} / 2^{n-1} \bmod 2=Z_{n}$.

\begin{tabular}{ccccccccccccccccc}
\hline$n$ & 0 & 1 & 2 & 3 & 4 & 5 & 6 & 7 & 8 & 9 & 10 & 11 & 12 & 13 & 14 & 15 \\
\hline$H_{n}$ & 1 & 1 & -2 & 0 & 0 & 0 & 0 & 64 & 128 & 0 & 0 & 0 & 0 & 0 & 0 & 0 \\
$u_{n}$ & 0 & 1 & 1 & 0 & 0 & 0 & 0 & 1 & 1 & 0 & 0 & 0 & 0 & 0 & 0 & 0 \\
\hline
\end{tabular}




\section{Application to Irrationality Exponent}

Our result about the automaticity of the sequence $H_{n} / 2^{n-1}(\bmod 2)$ enables us to obtain upper bounds of irrationality exponents of a family of automatic numbers. Let $\xi$ be an irrational real number. The irrationality exponent $\mu(\xi)$ of $\xi$ measures the approximation rate of $\xi$ by rationals. It is defined as the supremum of the real numbers $\mu$ such that the inequality

$$
\left|\xi-\frac{p}{q}\right|<\frac{1}{q^{\mu}}
$$

holds for infinitely many rational numbers $p / q$. In this section we will make use of the following method developed by Bugeaud et al. in 4 .

Theorem 4.1. Let $d \geq 2$ be an integer, and $\left(c_{j}\right)_{j \geq 0}$ be an integer sequence such that $f(z)=\sum_{j=0}^{\infty} c_{j} z^{j}$ converges inside the unit disk. Suppose that there exist integer polynomials $A(z), B(z), C(z)$ and $D(z)$ such that

$$
f(z)=\frac{A(z)}{B(z)}+\frac{C(z)}{D(z)} f\left(z^{d}\right) .
$$

Let $b \geq 2$ be an integer such that $B\left(\frac{1}{b^{d^{m}}}\right) C\left(\frac{1}{b^{d^{m}}}\right) D\left(\frac{1}{b^{d^{m}}}\right) \neq 0$ for all integers $m>0$. If there exists an increasing sequence of positive integers $\left(a_{i}\right)_{i \geq 0}$ such that $H_{a_{i}}(f) \neq$ 0 for all integers $i \geq 0$ and $\limsup \frac{a_{i+1}}{a_{i}}=\rho$, then $f(1 / b)$ is transcendental, and we have

$$
\mu\left(f\left(\frac{1}{b}\right)\right) \leq(1+\rho) \min \left\{\rho^{2}, d\right\} .
$$

In particular, the irrationality exponent of $f(1 / b)$ is equal to 2 if $\rho=1$.

A real number $\xi$ is said to be automatic if there exist two integers $d, b \geq 2$ such that the $b$-ary expansion of $\xi$ is $d$-automatic. We consider sequences of the form $\tilde{\mathbf{f}}=\Phi(\tilde{\mathbf{v}})$ defined in (1.2). This series satisfy the functional equation

$$
\tilde{\mathbf{f}}(x)=\tilde{\mathbf{v}}(x) \tilde{\mathbf{f}}\left(x^{d}\right) .
$$

As $\tilde{\mathbf{f}}$ takes only \pm 1 as coefficients, it converges inside the unit disk. Moreover, the polynomial $\tilde{\mathbf{v}}$ does not have any root of the form $1 / b^{d^{m}}$ for $b \geq 2$ and $m \geq 1$. This is because if we write $\tilde{\mathbf{v}}(x)=\sum_{i=0}^{d-1} v_{i} x^{i}$, then for any prime factor $p$ of $b$, the $p$-adic valuation of $\tilde{\mathbf{v}}\left(1 / b^{d^{m}}\right)$ is equal to the $p$-adic valuation of $\left(1 / b^{d^{m}}\right)^{d-1}$. So we may apply Theorem 4.1 to automatic real numbers $f\left(\frac{1}{b}\right):=\sum_{j=0}^{\infty} \frac{f_{j}}{b^{j}}$ for $b \geq 2$.

4.1. Two Examples. For the series $\tilde{\mathbf{f}}=\Phi(1,1,-1,-1,1)$ considered in Section 3.1. from relations between elements in the kernel of the sequence $\mathbf{Z}$, we deduce that the sequence $\left(Z_{n}\right)=\left(H_{n} / 2^{n-1}(\bmod 2)\right)$ is equal to $\tau\left(s^{\infty}(A)\right)$ where $s$ is the 5 -substitution

$$
\begin{aligned}
& A \mapsto A B C D A \\
& B \mapsto B C D A B \\
& C \mapsto C D D D D \\
& D \mapsto D D D D D,
\end{aligned}
$$


and $\tau$ is the coding $A, D \mapsto 0, B, C \mapsto 1$. Therefore,

$$
\mathbf{Z}=\tau\left(s^{n}(A) s^{n}(B) s^{n}(C) s^{n}(D) s^{n}(A) s^{n+1}(B) \ldots\right)
$$

for $n \geq 1$. We have $s^{n}(C)=C D^{5^{n}-1}, s^{n}(D)=D^{5^{n}}$, and $s^{n}(A)$ begins by $A B$. If we let $a_{n}$ denote the position of the $n$-th 1 in the sequence $\mathbf{Z}$, then is it easy to observe that $\lim \sup a_{i+1} / a_{i}$ is realized by the gap between the first letter in $s^{n}(C)$ and the second letter in the second $s^{n}(A)$. Therefore

$$
\rho=\limsup _{i \rightarrow \infty} \frac{a_{i+1}}{a_{i}}=\lim _{n \rightarrow \infty} \frac{5^{n} \times 4+1}{5^{n} \times 2}=2 .
$$

Theorem 4.1 then gives

$$
\mu(f(1 / b)) \leq 12
$$

for all integers $b \geq 2$.

For the series $\tilde{\mathbf{f}}=\Phi(1,1,-1,-1)$ considered in Section 3.2 from relations between elements in the kernel of the sequence $\mathbf{Z}$, we deduce that the sequence $\left(Z_{n}=\left(H_{n} / 2^{n-1}(\bmod 2)\right)\right.$ is equal to $\tau\left(s^{\infty}(A)\right)$ where $s$ is the 4-substitution

$$
\begin{aligned}
& A \mapsto A B C D \\
& B \mapsto D D A B \\
& C \mapsto C D D D \\
& D \mapsto D D D D,
\end{aligned}
$$

and $\tau$ is the coding $A, D \mapsto 0, B, C \mapsto 1$. Therefore,

$$
\mathbf{Z}=\tau\left(s^{n}(A) s^{n}(B) s^{n}(C) s^{n}(D) s^{n+1}(B) \ldots\right)
$$

for $n \geq 1$. We have $s^{n}(C)=C D^{4^{n}-1}, s^{n}(D)=D^{4^{n}}$, and $s^{n+1}(B)$ begins by $D^{4^{n} \times 2} A B$. We let $a_{n}$ denote the position of the $n$-th 1 in the sequence $\mathbf{Z}$. We observe that $\lim \sup a_{i+1} / a_{i}$ is realized by the gap between the first letter in $s^{n}(C)$ and the first $B$ in $s^{n+1}(B)$. Therefore

$$
\rho=\limsup _{i \rightarrow \infty} \frac{a_{i+1}}{a_{i}}=\lim _{n \rightarrow \infty} \frac{4^{n+1}+4^{n} \times 2+1}{4^{n} \times 2}=3 .
$$

Theorem 4.1 then gives

$$
\mu(f(1 / b)) \leq 16
$$

for all integers $b \geq 2$.

4.2. The General Case and Comparison with Previous Upper Bounds. To find an upper bound of the irrational exponent of an automatic number of the form $f(1 / b)$ where $\tilde{\mathbf{f}}=\Phi(\tilde{\mathbf{v}})$. We proceed as follows. First, find a substitution $s$ and a coding $\tau$ such that $\mathbf{Z}$ is the image by $\tau$ of a fixed point of $s$; then, calculate $\rho$ directly as in the examples; finally, derive the irrationality exponent using Theorem 4.1. The following theorem [9] garantees that it is always possible to find such $s$ and $\tau$.

Theorem 4.2 (Cobham). A sequence $\mathbf{u}$ is d-automatic if and only if it is the image of a fixed point of a d-substitution.

The alphabet of the substitution $s$ is called the internal alphabet of $\mathbf{u}$. The irrationality exponent of automatic sequences has been studied in [1], where Adamczewski et al. established the following theorem: 
Theorem 4.3. Let $d$ and $b$ be two integers at least equal to two and let $\mathbf{a}=\left(a_{n}\right)_{n \geq 0}$ be an infinite sequence generated by a d-automaton and with values in $\{0,1, \ldots, b-1\}$. Let $m$ be the cardinality of the d-kernel of the sequence $\mathbf{a}$ and let $c$ be the cardinality of the internal alphabet associated to a. Then, the irrationality exponent $\mu(\xi)$ of the real number

$$
\xi:=\sum_{n=0}^{\infty} \frac{a_{n}}{b_{n}}
$$

satisfies

$$
\mu(\xi) \leq c d\left(d^{m}+1\right) .
$$

For certain sequences of the form $\Phi(\tilde{\mathbf{v}})$, the Hankel determinant method yields a better upper bound than this general estimate. For example, for the sequence $\Phi(1,1,-1,-1)$ considered in Section 4.1, our computation gives $\mu(f(1 / b) \leq 16$ while Theorem 4.3 gives $\mu(f(1 / b)) \leq 136$; for the sequence $\Phi(1,1,-1,-1,1)$, our computation gives $\mu(f(1 / b)) \leq 12$ while Theorem 4.3 gives $\mu(f(1 / b)) \leq 260$. For the Thue-Morse sequence $\mathbf{t}$, Theorem 4.3 gives $\mu(t(1 / b)) \leq 20$ and an argument more adapted to this specific sequence in the same article gives $\mu(t(1 / b)) \leq 5$, while Hankel determinant method yields $\mu(t(1 / b))=2$, as shown by Bugeaud in [5].

\section{IMPLEMENTATION AND OUTPUTS}

Our main result Theorem 1.1 says that for each \pm 1 -vector $\mathbf{v}$ of length $d$, the sequence of the reduced Hankel determinants modulo 2 of the sequence $\Phi(\tilde{\mathbf{v}}(x))$ is $d$-automatic. Since the proof of the main theorem is constructive, we implement the method to find the minimal $d$-DFAO of the sequence $\left(H^{n} / 2^{n-1}(\bmod 2)\right)$. This is done by the Python programs Apwen2.py and ASHanke1.py. 1 We illustrate below the usage of these programs and their output for the two examples studied in Section 3 and Section 4.1

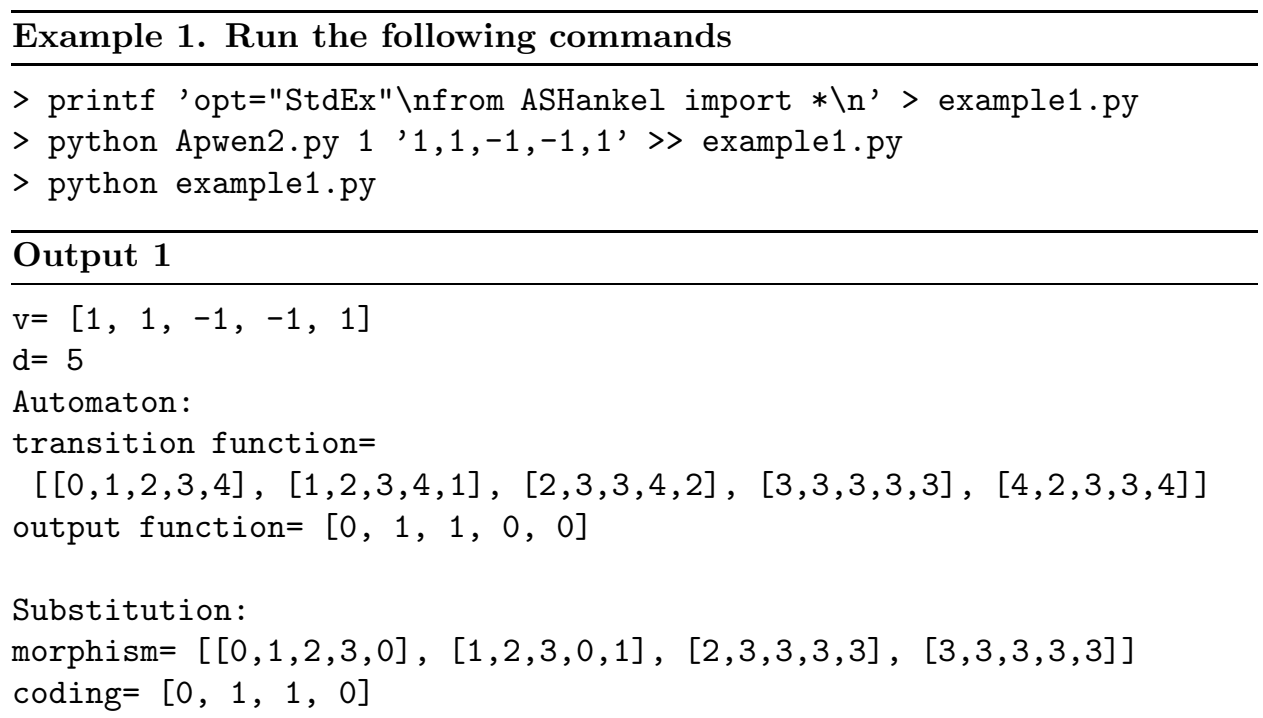

\footnotetext{
${ }^{1}$ The programs Apwen2.py and ASHankel.py and examples are available at irma.math.unistra.fr/ guoniu/papers/w03ashankel/
} 


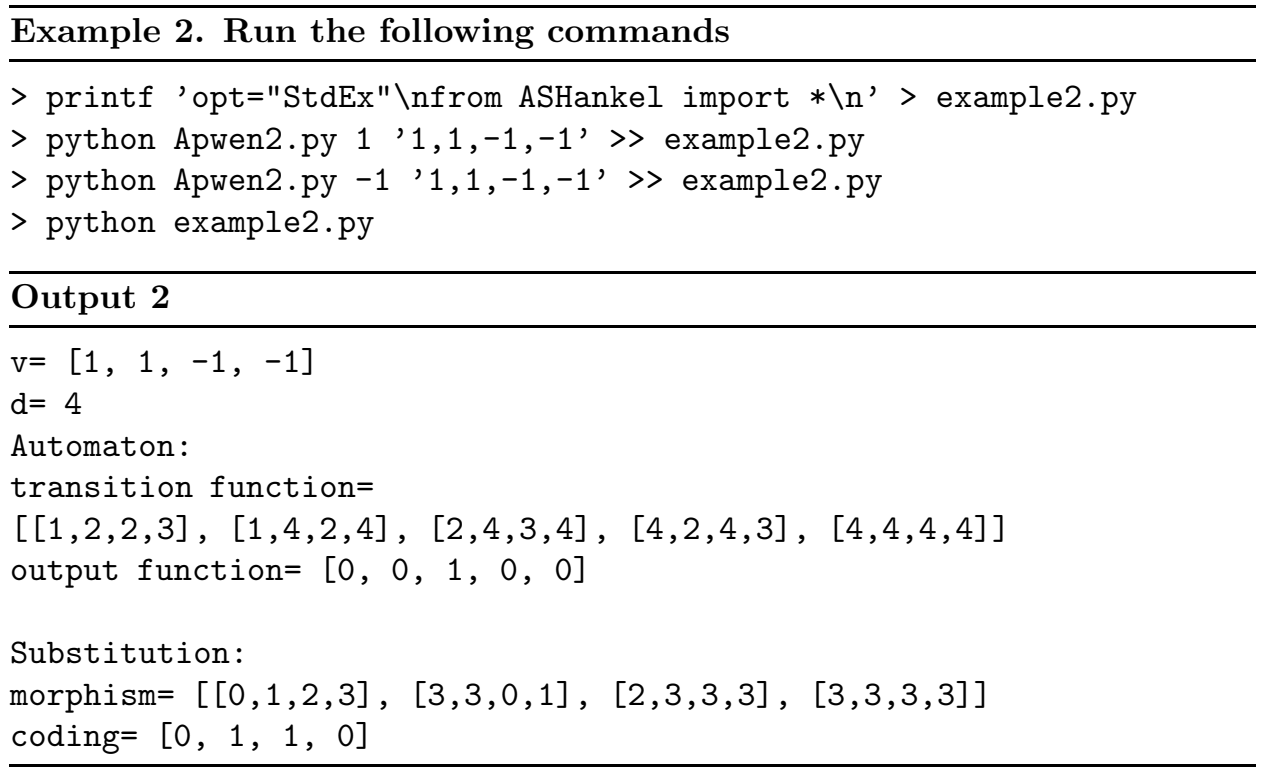

Acknowledgments. The authors would like to thank Zhi-Ying Wen and Hao $\mathrm{Wu}$ who invited them to Tsinghua University where the paper was finalized. The authors also thank Jean-Paul Allouche, Yann Bugeaud, Jacques Peyrière, Zhi-Xiong Wen, and Wen $\mathrm{Wu}$ for valuable discussions.

\section{REFERENCES}

[1] Boris Adamczewski and Julien Cassaigne. Diophantine properties of real numbers generated by finite automata. Compos. Math., 142(6):1351-1372, 2006.

[2] J.-P. Allouche, J. Peyrière, Z.-X. Wen, and Z.-Y Wen. Hankel determinants of the ThueMorse sequence. Ann. Inst. Fourier, Grenoble, 48:1-27, 1998.

[3] Jean-Paul Allouche and Jeffrey Shallit. Automatic sequences. Cambridge University Press, Cambridge, 2003. Theory, applications, generalizations.

[4] Y. Bugeaud, G.-N. Han, Wen Z.-Y., and Yao J.-Y. Hankel determinants, Padé approximations, and irrationality exponents. International Mathematics Research Notices (IMRN), 5:1467-1496, 2016.

[5] Yann Bugeaud. On the rational approximation to the Thue-Morse-Mahler numbers. Ann. Inst. Fourier (Grenoble), 61(5):2065-2076 (2012), 2011.

[6] Yann Bugeaud and Guo-Niu Han. A combinatorial proof of the non-vanishing of Hankel determinants of the Thue-Morse sequence. Electron. J. Combin., 21(3):Paper 3.26, 17, 2014.

[7] G. Christol, T. Kamae, M. Mendès France, and G. Rauzy. Suites algébriques, automates et substitutions. Bull. Soc. Math. France, 108(4):401-419, 1980.

[8] Gilles Christol. Ensembles presque periodiques $k$-reconnaissables. Theoret. Comput. Sci., 9(1):141-145, 1979.

[9] Alan Cobham. Uniform tag sequences. Math. Systems Theory, 6:164-192, 1972.

[10] M. Coons. On the rational approximation of the sum of the reciprocals of the fermat numbers. The Ramanujan Journal, 30(1):39-65, 2013.

[11] Samuel Eilenberg. Automata, languages, and machines. Vol. A. Academic Press [A subsidiary of Harcourt Brace Jovanovich, Publishers], New York, 1974. Pure and Applied Mathematics, Vol. 58.

[12] P. Flajolet. Combinatorial aspects of continued fractions. Discrete Math., 32(2):125-161, 1980.

[13] Robbert J. Fokkink, Cor Kraaikamp, and Jeffrey Shallit. Hankel matrices for the perioddoubling sequence. Indag. Math. (N.S.), 28(1):108-119, 2017. 
[14] Hao Fu and Guo-Niu Han. Computer assisted proof for Apwenian sequences. In Proceedings of the 2016 ACM International Symposium on Symbolic and Algebraic Computation, pages 231-238. ACM, New York, 2016.

[15] Hao Fu and Guo-Niu Han. Computer assisted proof for apwenian sequences related to Hankel determinants. arXiv, 1601.04370, 2016.

[16] Ying-Jun Guo, Zhi-Xiong Wen, and Wen Wu. On the irrationality exponent of the regular paperfolding numbers. Linear Algebra Appl., 446:237-264, 2014.

[17] Guo-Niu Han. Hankel determinant calculus for the Thue-Morse and related sequences. J. Number Theory, 147:374-395, 2015.

[18] Guo-Niu Han. Hankel continued fraction and its applications. Adv. Math., 303:295-321, 2016.

[19] Guo-Niu Han and Wen Wu. Evaluations of the Hankel determinants of a Thue-Morse-like sequence. Int. J. Number Theory, 11(6):1887-1904, 2015.

[20] C. Krattenthaler. Advanced determinant calculus. Sém. Lothar. Combin., 42:Art. B42q, 67 pp., 1999. http://www.mat.univie.ac.at/ ${ }^{\text {slc/ }}$

[21] C. Krattenthaler. Advanced determinant calculus: a complement. Linear Algebra Appl., 411:68-166, 2005.

[22] Raphaël Salem. Algebraic numbers and Fourier analysis. In Selected reprints, Wadsworth Math. Ser., pages iii+68. Wadsworth, Belmont, CA, 1983.

School of Mathematics and Statistics, Huazhong University of Science and TechNOLOGY, WuHAN, PR CHINA

E-mail address: huyining@protonmail.com

I.R.M.A., UMR 7501, Université de Strasbourg et CNRS, 7 rue René Descartes, F67084 Strasbourg, France

E-mail address: guoniu.han@unistra.fr 